\title{
Hysteroscopic assisted single-site robotic resection of cesarean scar defect (CSD): dual case reports and review of literature
}

\begin{abstract}
Background: The number of deliveries via cesarean section has increased in the United States, to a rate of $32.3 \%$, which is almost double the global rate of $18.6 \%$. With a greater rate of deliveries via cesarean section comes an increased rate of associated complications in subsequent pregnancies and longer hospital stays. One complication of cesarean section is the formation of a cesarean scar defect (CSD), niche or isthmocele which has no standard definition but can be grossly described as a disruption or defect in the myometrium associated with uterine scar. Approximately $1.9 \%$ of women are diagnosed with CSD; however the prevalence of CSD is difficult to quantify, given that smaller CSDs may be asymptomatic. As more women are encouraged towards trial of labor after cesarean (TOLAC), the performance of the uterus during labor is of growing concern due to the risk of uterine rupture.
\end{abstract}

We present two cases ${ }^{1}$ of Cesarean Scar Defect (CSD) repaired by hysteroscopy and roboticassisted single-incision laparoscopic surgery (RA-SILS) for cesarean scar resection.

Conclusion: Rising rates of Cesarean sections bring increased rates of complications, including infertility and pain. Fortunately, CSD can be repaired surgically with great success. With technological advances, MIGS has become the standard of care for many gynecologic surgeries, showing improved patient outcomes. There continues to be some debate over the efficacy of improved patient outcomes with robotic systems. However, these questions are often related to surgeon experience and surgical time. We have presented the first cases of CSD repair using RA-SILS assisted by hysteroscopy. More quantitative studies with specific measures are needed to fully understand the impact of minimally invasive gynecologic surgery and RA-SILS for CSD.

Keywords: cesarean scar defect, cesarean scar dehiscence, cesarean scar diverticulum, hysteroscopic assisted, hysteroscopy, isthmocele, isthmoplasty, laparoscopy, resection, niche, laparoendoscopic single-site surgery, residual myometrial thickness, single-incision laparoscopic surgery, transvaginal repair, uterine scar dehiscence

\footnotetext{
${ }^{1}$ A thorough case report search performed in early 2018 of Google Scholar, Pubmed, Medline, BMJ Case Reports, Wiley Online Library, and Oxford Medical Case Reports using keywords: CSD, Cesarean Scar Defect; Cesarean Scar Dehiscence; Cesarean Scar Diverticulum; Isthmocele; Isthmoplasty; Laparoscopy; Previous Cesarean Scar Defect; Niche; LESS, Laparoendoscopic Single-Site Surgery; SILS, Single-Incision Laparoscopic Surgery; Uterine Scar Dehiscence. The search produced no reported cases of CSD surgical repair using robotic single incision laparoscopic surgery with hysteroscopy.
}

Volume 9 Issue 4 - 2018

\section{Yiming Zhang, ${ }^{3}$ Shadi Rezai, ${ }^{1,6}$ Alexander C Hughes, ${ }^{2}$ Juan Saucedo, ${ }^{2}$ Ninad M Patil, ${ }^{4}$ Elise Bardawil,6 Cassandra E Henderson, ${ }^{5}$ Xiaoming Guan ${ }^{6}$}

'Department of Obstetrics and Gynecology, Southern California Kaiser Permanente, USA

${ }^{2}$ St. George's University, School of Medicine, St. George's, Grenada

${ }^{3}$ Division of Reproductive Medicine, Jinan Central Hospital Group, China

${ }^{4}$ Department of Pathology \& Immunology, Baylor College of Medicine, USA

${ }^{5}$ Maternal and Fetal Medicine, Department of Obstetrics and Gynecology, Lincoln Medical and Mental Health Center, USA ${ }^{6}$ Division of Minimally Invasive Gynecologic Surgery, Department of Obstetrics and Gynecology, Baylor College of Medicine, USA

Correspondence: Xiaoming Guan MD PhD, Section Chief and Fellowship Director, Division of Minimally Invasive Gynecologic Surgery, Department of Obstetrics and Gynecology, Baylor College of Medicine, 665I Main Street, I0th Floor, Houston, Texas, 77030, USA, Tel (832) 826-7464, Fax (832) 825-9349, Email xiaoming@bcm.edu

Received: June 12, 2018 | Published: July 25, 2018
Abbreviations: CSD, cesarean scar defect; TOLAC, trial of labor after cesarean; RA-SILS, robotic-assisted single-incision laparoscopic surgery; REI, reproductive endocrinology and infertility; HSG, hysterosalpingogram; AUB, abnormal uterine bleeding; MIGS, minimally invasive gynecological surgery

\section{Background}

The number of deliveries via cesarean section has increased in the United States, to a rate of $32.3 \%$, which is almost double the global rate of $18.6 \% .{ }^{1}$ With a greater rate of deliveries via cesarean section comes increased rate of associated complications in subsequent pregnancies and longer hospital stays. ${ }^{2}$ One complication of cesarean section is the formation of a cesarean scar defect (CSD), niche or isthmocele, which has no standard definition but can be grossly described as a disruption or defect in the myometrium associated with uterine scar. ${ }^{3-6}$
Approximately $1.9 \%$ of women are diagnosed with CSD, however the prevalence of CSD is difficult to quantify, given that smaller CSDs may be asymptomatic. ${ }^{4}$ As more women are encouraged towards a trial of labor after cesarean (TOLAC) the performance of the uterus during labor is of growing concern due to the risk of uterine rupture. ${ }^{2,7}$

Risk factors for CSD include cesarean section during advanced stage of labor, multiple cesarean deliveries, retroflexed uterus, and uterine incision nears the cervix., ${ }^{4,-11,12}$ Single-layer uterine closure has also been proposed as a risk factor for CSD, but there is still no consensus on the optimal approach to uterine closure. ${ }^{13,14}$ Small asymptomatic defects may not require treatment; however larger defects may cause pelvic pain, dysmenorrhea, intermenstrual bleeding or infertility, requiring surgical intervention. ${ }^{8,15}$ Surgical repair has shown to be an effective treatment, providing symptom relief for most patients and resolving infertility in $92 \%$ of patients. ${ }^{8,11,16}$ 
Options for repair include a laparoscopic, or vaginal approach, a multiport robotics procedure, and now, a single incision robotic laparoscopy. ${ }^{4,17-20}$ Minimally invasive approaches, such as robotic surgeries, have been shown by some studies to improve patient outcomes in gynecological surgery patients. ${ }^{21}$ We present two cases of Cesarean Scar Defect (CSD) repaired by hysteroscopy and roboticassisted single-incision laparoscopic surgery (RA-SILS) for cesarean scar resection.

\section{Presentation of case I}

The patient, a 34 year old gravida 2, para 2002 with a history of two previous cesarean deliveries, was referred to our clinic for evaluation of a possible CSD by her reproductive endocrinology and infertility (REI) specialist. Initially, she complained of dysmenorrhea, menorrhagia and dyspareunia, followed by a failed intrauterine insemination. The patient reported normal menstrual cycles prior to her first cesarean delivery in 2012. Subsequently, her symptoms gradually worsened, especially after the $2^{\text {nd }}$ cesarean delivery in 2014. Past medical history was unremarkable except for a case of rhabdomyolysis in August of 2017.

The REI workup included ultrasound, hysterosalpingogram (HSG), MRI and hysteroscopy with lysis of adhesions, and endometrial sampling for evaluation of failed intrauterine insemination, 6 months prior to referral. The initial ultrasound showed mucus accumulation and free fluid in the uterus (Figure 1). Hysteroscopy revealed a small out-pouching in the lower anterior uterine segment. A subsequent MRI in our clinic confirmed both the ultrasound and Hysteroscopic findings, revealing a small CSD, (Figure 2) for which she desired surgical correction.

After a discussion of the treatment options, the patient underwent CSD repair via hysteroscopy and RA-SILS for cesarean scar resection. The post-operative period was uneventful and the patient went home the same day. When the patient was followed up in the clinic, she denied any postoperative complications, resolution of vaginal mucus discharge with continued menorrhagia.

The CSD resection specimen collected intraoperatively was sent for pathologic examination and showed hyalinized and fibrotic tissue with chronic inflammation, consistent with old cesarean scar (Figure $3)$.
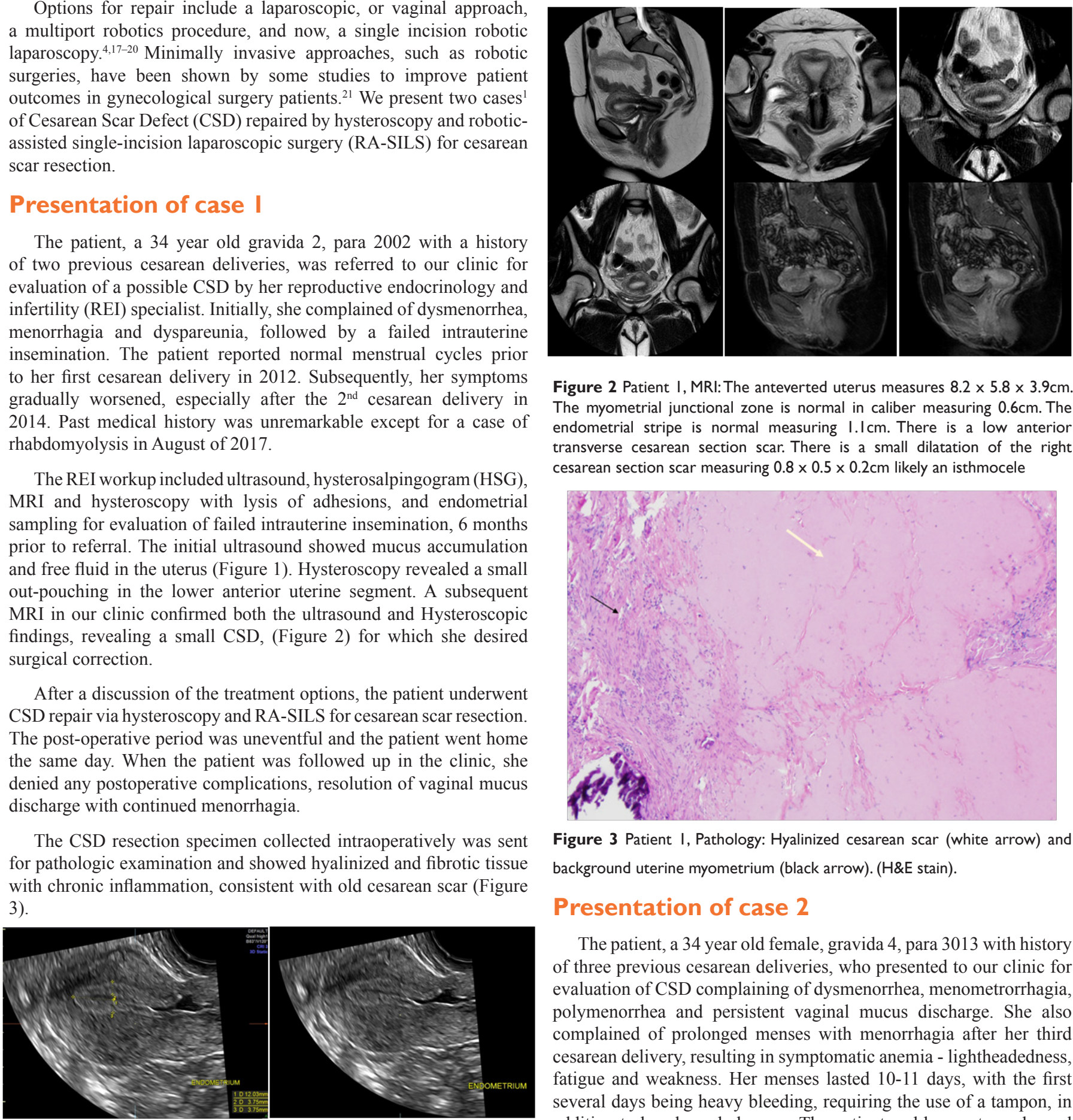

Figure 2 Patient I, MRI:The anteverted uterus measures $8.2 \times 5.8 \times 3.9 \mathrm{~cm}$ The myometrial junctional zone is normal in caliber measuring $0.6 \mathrm{~cm}$. The endometrial stripe is normal measuring $1.1 \mathrm{~cm}$. There is a low anterior transverse cesarean section scar. There is a small dilatation of the right cesarean section scar measuring $0.8 \times 0.5 \times 0.2 \mathrm{~cm}$ likely an isthmocele

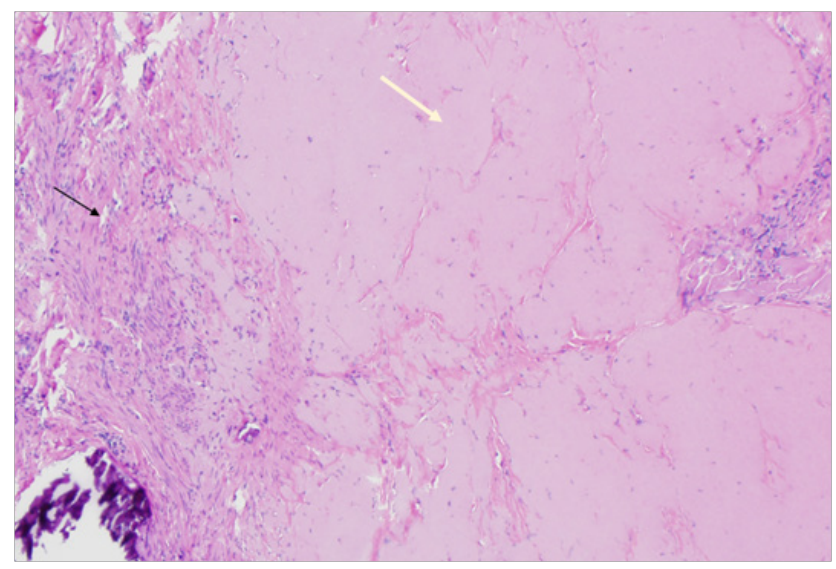

Figure 3 Patient I, Pathology: Hyalinized cesarean scar (white arrow) and background uterine myometrium (black arrow). (H\&E stain).

\section{Presentation of case 2}

The patient, a 34 year old female, gravida 4, para 3013 with history of three previous cesarean deliveries, who presented to our clinic for evaluation of CSD complaining of dysmenorrhea, menometrorrhagia, polymenorrhea and persistent vaginal mucus discharge. She also complained of prolonged menses with menorrhagia after her third cesarean delivery, resulting in symptomatic anemia - lightheadedness, fatigue and weakness. Her menses lasted 10-11 days, with the first several days being heavy bleeding, requiring the use of a tampon, in addition to hourly pad changes. The patient and her partner planned to conceive but desired surgical repair of her symptomatic CSD prior to conceiving.

I Patient I, Ultrasound: Ultrasound on $6 / 3 / 17$, showing accumulation and free fluid in the uterus as well as a scar defect in anterio uterine wall (Red Circle).

${ }^{2} \mathrm{~A}$ thorough case report search performed in early 2018 of Google Scholar, Pubmed, Medline, BMJ Case Reports, Wiley Online Library, and Oxford Medical Case Reports using keywords: CSD, Cesarean Scar Defect; Cesarean Scar Dehiscence; Cesarean Scar Diverticulum; Isthmocele; Isthmoplasty; Laparoscopy; Previous Cesarean Scar Defect; Niche; LESS, Laparoendoscopic Single-Site Surgery; SILS, Single-Incision Laparoscopic Surgery; Uterine Scar Dehiscence. The search produced no reported cases of CSD surgical repair using robotic single incision laparoscopic surgery with hysteroscopy.

Pelvic ultrasound revealed evidence of cesarean scar defect (Figure 4). After counseling, the patient wished to proceed with an operative repair therefore underwent hysteroscopy, RA-SILS repair and resection of the CSD, lysis of adhesions (salpingolysis, ovariolysis, and lysis of adnexal adhesions).

Hysteroscopy in the operating room revealed anterior CSD cephalad to the internal cervical os (Figure 5), an unremarkable uterine cavity, and bilateral ostia. Laparoscopy revealed dense adhesions of 
the bladder to the anterior lower uterine segment. The adhesions were lysed allowing for greater mobilization of the uterus. The CSD was excised and repaired. The post-operative period was uneventful, and the patient went home the same day.

The patient was counseled about the potential for new defect occurring during her next pregnancy and/or after her next cesarean delivery, and a minimum delay of two months was recommended before conceiving to allow for healing of the uterus and umbilical incision.

The CSD resection specimen was sent for pathologic examination which showed fragments of endomyometrial tissue with scarring consistent with the cesarean scar, along with old placental side nodule with dystrophic calcifications (Figure 6) (Figure 7).

When the patient was followed up three weeks post-operatively, she reported improved symptoms - lighter menstrual flow, significantly less pain and resolution of her vaginal mucous discharge.
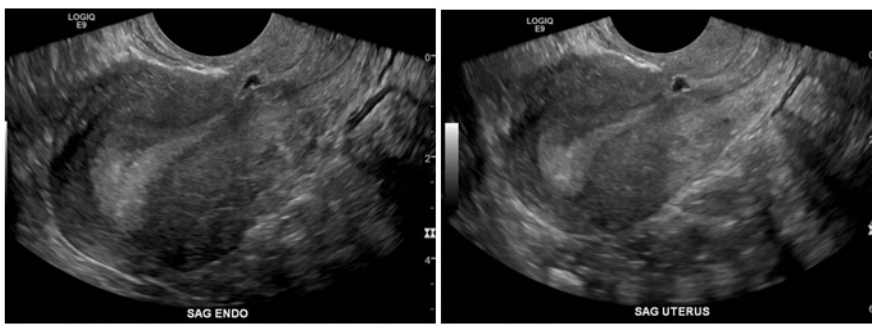

Figure 4 Patient 2, Ultrasound: 5/23/I7, showing evidence of dehiscence.

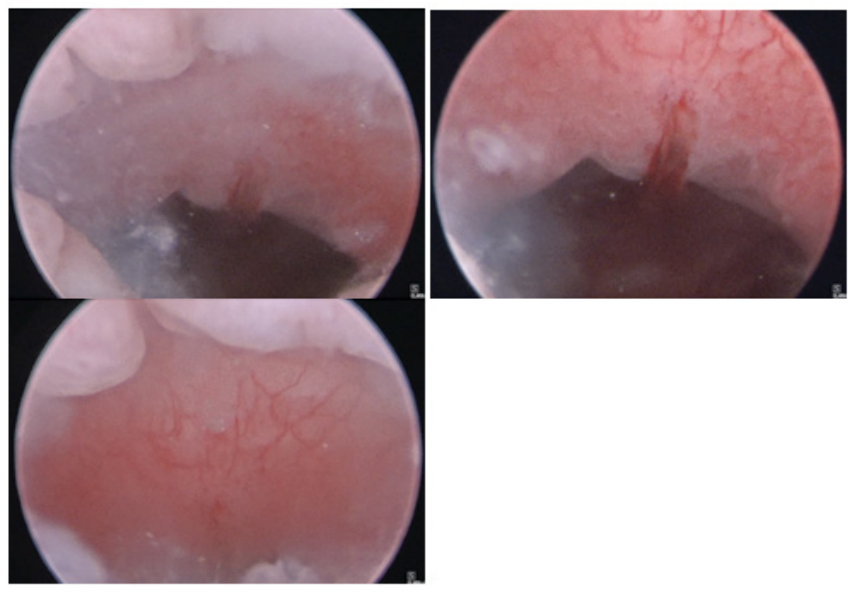

Figure 5 Patient 2, Hysteroscopic images: Hysteroscopy showing anterior CSD cephalad to the internal cervical os, with an unremarkable uterine cavity.

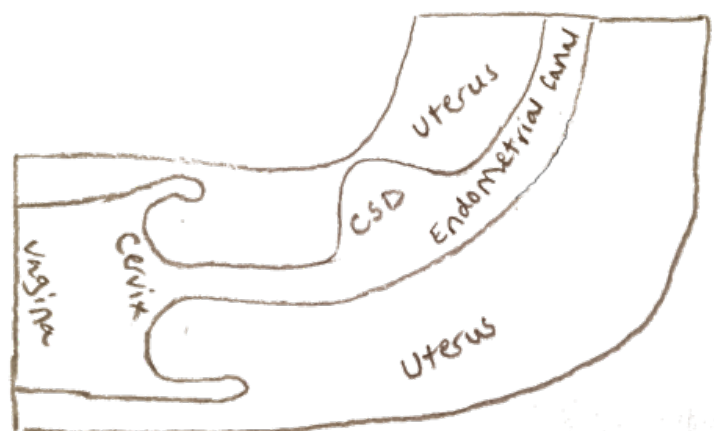

Figure 6 Schematic diagram of a niche at the site of the caesarean section scar.

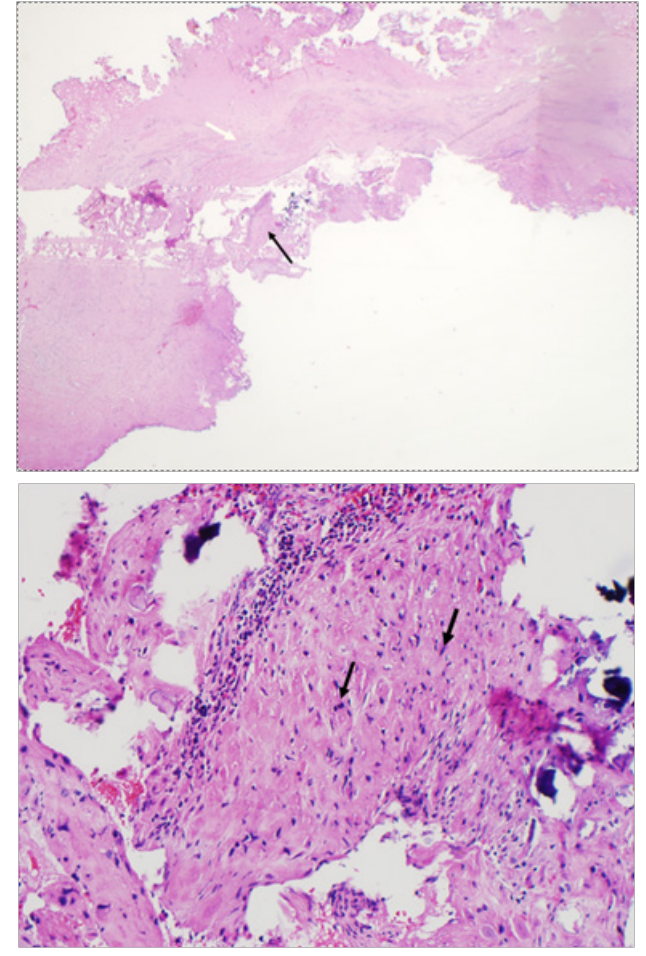

Figure 7 Patient 2, Pathology slides: a. The pathology figure 7a would roughly correspond to the red circle in figure 6: Low power view of resected fibrotic cesarean scar (white arrow) with placental site nodule (black arrow). (H\&E stain) b. High Power view of placental site nodule (black arrow) showing hyalinization with degenerative implantation site trophoblast. (H\&E stain).

\section{Discussion}

CSD was initially described in 1975 by Stewart and Fader relating to abnormal uterine bleeding (AUB) and pelvic pain. ${ }^{22}$ More recently, it has been associated with infertility and miscarriage. ${ }^{21,23,24}$ Though the association with infertility is clear, there exists no consensus for the diagnosis of infertility secondary to CSD. ${ }^{24}$ In 2015, Tanimura et al. ${ }^{21}$ proposed diagnostic criteria for secondary infertility due to CSD (Table 1); while Schepker et al. ${ }^{25}$ proposed the possibility of CSD as a clinical diagnosis based on reported symptoms.

Table I Proposed diagnostic criteria for infertility secondary to CSD (I8)

\section{S. No Proposed diagnostic criteria}

Retention of blood in the uterine scar or the uterine cavity during the period from the end of menstruation to ovulation

2

Unsuccessful attempts to become pregnant after two or more procedures of artificial insemination or due to other unknown cause of infertility

The current treatment of choice for symptomatic CSD is surgical excision of the defect, though guidelines do not suggest a preferred approach. ${ }^{3-5}$ As previously stated, surgical options include a laparoscopic, vaginal, robotics and RA-SILS, with or without hysteroscopy., ${ }^{4,17,18,22,23} \mathrm{Li}$ et al. ${ }^{26}$ found the combined hysteroscopic/ laparoscopic repair showed superior patient outcomes compared to a transvaginal repair alone. The Li et al. ${ }^{26}$ study used the hysteroscope intraoperatively to directly evaluate the scar and delineate it using transillumination. 
RA-SILS was used in both cases and both were initiated by making a single incision at the umbilicus followed by the introduction of a single incision laparoscopic port/device. The port was large enough to accommodate multiple instruments while maintaining an elevated intraabdominal pressure. The issue of triangulation was avoided using a curved robotic instrument to reestablish angles needed for effective triangulation. In the cases presented, a hysteroscope was used before docking the robot, to delineate the scar for visualization on laparoscopy. This technique was successful in the first case but failed for the second case due to extensive adhesions. In this second case, once the adhesions were lysed and a bladder flap was formed, visualization was successful. After complete excision of the scars, in both cases, via monopolar hook electrode, the new uterine incision was closed with 2 layers of v-locked sutures. During their first followup visits, both patients described resolution of symptoms and were counseled to avoid getting pregnant for at least the first two months postoperatively.

Two newer minimally invasive gynecological surgery (MIGS) techniques were utilized in combination to repair CSD. Over the last 8 years, there have been multiple cases of traditional multiport robotic laparoscopic CSD repairs. ${ }^{4,27,28}$ The RA-SILS technique has been used successfully for benign myomectomy surgery but there have been no previous published reports of single site robotic repair of CSD. ${ }^{29}$

Stewart et al. ${ }^{22}$ found that the use of SILS, when compared to traditional multiport laparoscopic surgery, showed improved patient outcomes. Multiple studies have shown SILS surgery decreases postoperative pain and use of pain medication. ${ }^{22}$ Other proposed outcomes which were not studied include enhanced cosmetics and incisional morbidity. The use of robotics, by an experienced surgeon, has shown improved outcomes with reduced surgical time, reducing hospital stays and improving patient outcomes in general..$^{30} \mathrm{~A}$ specific study and review of outcomes relating the use of SILS or RA-SILS for CSD repair has yet to be done.

\section{Conclusion}

Rising rates of Cesarean sections bring increased rates of complications, including infertility and pain. ${ }^{1}$ Fortunately, CSD can be repaired surgically with great success. With technological advances, MIGS has become the standard of care for many gynecologic surgeries, showing improved patient outcomes. There continues to be some debate over the efficacy of improved patient outcomes with robotic systems. However, these questions are often related to surgeon experience and surgical time.

Now that robotics assisted surgeries are being introduced earlier in residency training, robotic surgical times will no doubt improve. Even though some question if robotics and single site surgical systems are beneficial, one cannot deny that improved laparoscopic dexterity and single small incisions should improve patient outcomes. We have presented two cases of CSD repair using RA-SILS assisted by hysteroscopy. More quantitative studies with specific measures are needed to fully understand the impact of MIGS and RA-SILS for CSD.

\section{Acknowledgments}

Dr. Xiaoming Guan is a speaker for Applied Medical, Rancho Santa Margarita, and California.

\section{Conflicts of interest}

Authors did not report any potential conflicts of interests.

\section{References}

1. Betrán AP, Ye $\mathrm{J}$, Moller $\mathrm{AB}$, et al. The increasing trend in caesarean section rates: global, regional and national estimates: 1990-2014. PLoS One. 2016;11(2): 0148343

2. Raimondo G, Grifone G, Raimondo D, et al. Hysteroscopic treatment of symptomatic cesarean-induced isthmocele: a prospective study. J Minim Invasive Gynecol. 2015;22(2):297-301.

3. Bharatam KK. Cesarean section uterine scar dehiscence - A review. Uterus \& Ovary. 2015;2:e751.

4. La Rosa MF, McCarthy S, Richter C, et al. Robotic repair of uterine dehiscence. JSLS. 2013; 17(1):156-60.

5. Bharatam KK, Sivaraja PK, Abineshwar NJ, et al. The tip of the iceberg: Post caesarean wound dehiscence presenting as abdominal wound sepsis. Int J Surg Case Rep. 2015;9:69-71.

6. Monteagudo A, Carreno C, Timor-Tritsch IE, Saline infusion sonohysterography in non pregnant women with previous cesarean delivery: the "niche" in the scar. J Ultrasound Med. 2001;20(10):1105-15.

7. Naji O, Abdallah Y, Bij De Vaate AJ, et al. Standardized approach for imaging and measuring Cesarean section scars using ultrasonography. Ultrasound Obstet Gynecol. 2012;39(3):252-9.

8. Api M, Boza A, Gorgen H, et al. Should cesarean scar defect be treated laparoscopically? a case report and review of the literature. J Minim Invasive Gynecol. 2015;22(7):1145-52.

9. Vikhareva Osser O, Valentin L. Risk factors for incomplete healing of the uterine incision after caesarean section. BJOG. 2010;117(9):1119-26.

10. Donnez O, Jadoul P, Squifflet J, et al. Laparoscopic repair of wide and deep uterine scar dehiscence after cesarean section. Fertil Steril. 2008;89(4):974-80.

11. Xu HY, Yang MY, Zhang X, et al. Efficacy of caesarean scar defect repair in improving postmenstrual bleeding and factors associated with poor effect. J Obstet Gynaecol. 2017;37(8):1076-1081.

12. Wang CB, Chiu WW, Lee CY, et al. Cesarean scar defect: correlation between Cesarean section number, defect size, clinical symptoms and uterine position. Ultrasound Obstet Gynecol. 2009;34(1):85-9.

13. Di Spiezio Sardo A, Saccone G, McCurdy R, et al. Risk of Cesarean scar defect following single- vs double-layer uterine closure: systematic review and meta-analysis of randomized controlled trials. Ultrasound Obstet Gynecol. 2017;50(5):578-583.

14. Roberge S, Demers S, Berghella V, et al. Impact of single- vs double-layer closure on adverse outcomes and uterine scar defect: a systematic review and meta-analysis. Am J Obstet Gynecol. 2014;211(5):453-60.

15. Erickson SS, Van Voorhis BJ. Intermenstrual bleeding secondary to cesarean scar diverticuli: report of three cases. Obstet Gynecol. 1999;93(5 Pt 2):802-5.

16. Bakavičiūtė $G$, Špiliauskaitė S, Meškauskienė A, et al. Laparoscopic repair of the uterine scar defect - successful treatment of secondary infertility: a case report and literature review. Acta Med Litu. 2016;23(4):227-231.

17. Vervoort A, van der Voet LF, Hehenkamp W, et al. Hysteroscopic resection of a uterine caesarean scar defect (niche) in women with postmenstrual spotting: a randomized controlled trial. BJOG. 2018;125(3):326-334. 
18. Zhang Y. A Comparative study of transvaginal repair and laparoscopic repair in the management of patients with previous cesarean scar defect. $J$ Minim Invasive Gynecol. 2016;23(4):535-41.

19. Luo L, Niu G, Wang Q, et al. Vaginal repair of cesarean section scar diverticula. J Minim Invasive Gynecol. 2012;19(4):454-8.

20. Chen Y, Chang Y, Yao S. Transvaginal management of cesarean scar section diverticulum: a novel surgical treatment, Med Sci Monit. 2014;20:1395-9.

21. Tanimura S, Funamoto H, Hosono T, et al. New diagnostic criteria and operative strategy for cesarean scar syndrome: Endoscopic repair for secondary infertility caused by cesarean scar defect. J Obstet Gynaecol Res. 2015;41(9):1363-9.

22. Stewart KS, Evans TW. Recurrent bleeding from the lower segmen scar--a late complication of Caesarean section. Br J Obstet Gynaecol. 1975;82(8):682-6.

23. Van der Voet LF, Vervoort AJ, Veersema S, et al. Minimally invasive therapy for gynecological symptoms related to a niche in the caesarean scar: a systematic review. BJOG. 2014;121(2):145-56.

24. Bij de Vaate AJ, van der Voet LF, Naji O, et al. Prevalence, potential risk factors for development and symptoms related to the presence of uterine niches following Cesarean section: systematic review. Ultrasound Obstet Gynecol. 2014;43(4):372-82.
25. Schepker N, Garcia-Rocha GJ, von Versen-Höynck F, et al. Clinical diagnosis and therapy of uterine scar defects after caesarean section in non-pregnant women. Arch Gynecol Obstet. 2015;291(6):1417-23.

26. Li C, Tang S, Gao X, et al. Efficacy of combined laparoscopic and hysteroscopic repair of post-cesarean section uterine diverticulum: a retrospective analysis. Biomed Res Int. 2016;2016:1765624.

27. Yalcinkaya TM, Akar ME, Kammire LD, et al. Robotic-assisted laparoscopic repair of symptomatic cesarean scar defect: a report of two cases. J Reprod Med. 2011;56(5-6):265-70.

28. Mahmoud MS, Nezhat FR. Robotic-assisted laparoscopic repair of a cesarean section scar defect. J Minim Invasive Gynecol. 2015;22(7):11356.

29. Choi EJ, Rho AM, Lee SR, et al. Robotic single-site myomectomy: clinical analysis of 61 consecutive cases. J Minim Invasive Gynecol. 2017;24(4):632-639.

30. Shashoua AR, Gill D, Locher SR. Robotic-assisted total laparoscopic hysterectomy versus conventional total laparoscopic hysterectomy. JSLS. 2009;13(3):364-9. 